\title{
From Catch-all to Clarity: Revising a First-Year, Multidisciplinary Introduc- tory Course
}

\section{Dr. Tanya Kunberger, Florida Gulf Coast University}

Dr. Kunberger is an Associate Professor in the Department of Environmental and Civil Engineering in the U. A. Whitaker College of Engineering at Florida Gulf Coast University. Dr. Kunberger received her B.C.E. and certificate in Geochemistry from the Georgia Institute of Technology and her M.S. and Ph.D. in Civil Engineering with a minor in Soil Science from North Carolina State University. Her areas of specialization are geotechnical and geo-environmental engineering. Educational areas of interest are selfefficacy and persistence in engineering and development of an interest in STEM topics in K-12 students.

\section{Dr. Chris Geiger, Florida Gulf Coast University}

Chris Geiger is an Associate Professor and Chair of the Department of Bioengineering in the U.A.Whitaker College of Engineering at Florida Gulf Coast University. He received his M.S and Ph.D.degrees in Biomedical Engineering from Northwestern University in 1999 and 2003, respectively,and his B.S. in Chemical Engineering from Northwestern University in 1996. 


\section{From Catch-all to Clarity: Revising a First-Year, Multidisciplinary Introductory Course}

\section{Introduction and History of Florida Gulf Coast University}

The National Resource Center for The First-Year Experience and Students in Transition describes South Carolina's University 101 course, which was first taught in 1972 as the impetus to improve educational experiences for first year students ${ }^{\mathrm{a}}$. By 1982, over 175 educators across the country came together to discuss first-year seminars, and the following year the Annual Conference on the Freshman Year Experience was born. Today, an effective first-year experience has been identified as a high impact educational practice by the Association of American Colleges and Universities (AAC\&U). Although these experiences differ significantly from university to university, ranging anywhere from a single course specifically taken in the major itself, through more involved practices including live-learn communities, Kuh emphasizes the most influential points of a first-year experience include a "strong emphasis on critical inquiry, frequent writing, information literacy, [and] collaborative learning." Faculty at Florida Gulf Coast University (FGCU) set out to improve their gateway course to the engineering curriculum, a one-credit hour course common across three of the four programs within the U. A. Whitaker College of Engineering, being mindful not only of including identified high impact educational practices, but also incorporating the University's upcoming 5-year Quality Enhancement Plan (QEP), which focuses on "improving student learning in relation to Writing, Critical Thinking, and Information Literacy."b The result of these efforts is a course with an emphasis on the development of information literacy, teamwork, and communication skills, focusing on engineering innovations related to the Grand Challenges. This paper will present the development of course expectations and ties to existing best practices, the revised course layout, student assessment and feedback of the initial offering, and instructor perspectives of the revised course.

FGCU first opened its doors to students in Fall 1997 as the tenth University within the Florida state system. FGCU is predominantly an undergraduate institution, with over $90 \%$ of its over 14,000 total students enrolled at the undergraduate level. Due in part to being the largest metropolitan region without an accredited engineering program ${ }^{2}$, the U.A. Whitaker School of Engineering (WSOE) was established in 2004, and classes began in the fall of 2005, with degrees offered in Civil, Environmental and Bioengineering. The mission of the WSOE was to be "Internationally recognized for excellence in interdisciplinary engineering education", and to incorporate innovative, interdisciplinary methods to our engineering classes ${ }^{2}$. Faculty embraced the recommendations of the National Academy of Engineering's Engineer of 2020 initiative $^{3,4}$ in designing the courses, curricula, and overall undergraduate engineering experience within the School. In addition to the interdisciplinary nature of the program's beginnings, the classes were designed to be taught within an integrated lecture/lab environment with extended contact time,

\footnotetext{
${ }^{a}$ http://www.sc.edu/fye/center/history.html

${ }^{b}$ http://www.fgcu.edu/qep/
} 
such that a three-credit class would meet for four hours over two days, allowing for unique opportunities in curriculum development. Studio classrooms based on the SCALE-UP (Student Centered Active Learning Environment for Undergraduate Programs) $\operatorname{model}^{5}$ were designed in Holmes Hall to accommodate this model of engineering education. In Fall 2011, the U.A. Whitaker School of Engineering became the U.A. Whitaker College of Engineering (WCE), and today the college has a student enrollment of almost 900, with over 1000 students projected by this fall.

\section{The Introductory Engineering Course - Fall 2005 - Spring 2014}

"Introduction to the Engineering Profession" is a one-credit hour course that was originally developed to provide an overview of the different engineering programs at FGCU. This overview was designed to give students a better understanding not only of the various majors, but also what opportunities and responsibilities a career in engineering would have, during their college years as well as post-graduation. As a new engineering school at a new University, the first offerings of this course were also designed to encourage students to consider engineering as a preferred career choice. As the course evolved and matured, additional information was added to the curriculum based on topics identified by faculty as necessary skills for engineering majors, including topics such as unit conversions, communication skills, resume building, team-work, and presentations by individuals working in industry. Since topics were added over the span of a decade, often times without other material being removed, each new concept was treated more as a module, rather than being fully integrated into the course, and the result became a catch-all type course with little topical continuity from week to week. Furthermore, the workload for both the faculty and students became significantly greater than what would reasonably be expected of a course with a one-credit hour designation.

Figure 1 shows the course learning outcomes from the previous version of the course (comparing them to the revised course learning outcome), and Figure 2 shows how the course evolved from 2009 to 2013, with the addition of a new program (Software Engineering), and two lessons on units and unit conversions, replacing modules on student success and the engineering profession. The typical structure of any given lesson was to have the students read the assigned reading and take an online reading quiz prior to coming to class, have a brief lesson / discussion on that topic at the beginning of class, followed by a "Class Activity" which could be an internal or external speaker, a team-building activity related to the agenda topic, or time to work on upcoming deliverables regarding the final project, which for many of the offerings was the development of a Rube Goldberg machine using a series of simple machines. Although the class claimed to be built around "active learning concepts", as one can see by 2013, very little "active learning concepts" had survived, and many of the lessons had evolved to having fewer and fewer activities and more and more speakers. There were many reasons for this shift, but it ultimately boiled down to time, resources, and learning gains. While the interactive in-class activities and the student-driven nature of the semester projects were enjoyed by the students, the learning gains associated with both the in-class activities and the semester project were viewed as being minimal or inadequate, and did not provide the students with insight into or preparation for the 
work expectations associated with future engineering courses. As shown in Figure 3, even though changes were made to the course on an annual basis, from 2010 to 2013, few graduating seniors found the introductory course to be useful.

\begin{tabular}{|c|c|}
\hline Previous Course Learning Objectives & New Course Learning Objectives \\
\hline $\begin{array}{l}\text { At the conclusion of this course, students will be able } \\
\text { to: } \\
\text { 1. Compare and contrast the engineering disciplines } \\
\text { offered at FGCU; } \\
\text { 2. Be familiar with and be able to apply the engineering } \\
\text { design process; } \\
\text { 3. Work on a team effectively to solve problems, } \\
\text { complete projects, and make presentations; } \\
\text { 4. Design and assemble simple projects; } \\
\text { 5. Explain the importance of having high ethical } \\
\text { standards; } \\
\text { 6. Explain the characteristics of effective team behavior } \\
\text { and the importance of teamwork in an engineering } \\
\text { environment; } \\
\text { 7. Explain the importance of professionalism; } \\
\text { 8. Explain the importance of having good time } \\
\text { management and study skills; and } \\
\text { 9. Specify the necessary courses and course sequence } \\
\text { needed to graduate in a timely manner in the } \\
\text { engineering discipline of choice }\end{array}$ & $\begin{array}{l}\text { At the completion of the course, students will be able } \\
\text { to: } \\
\text { - Summarize and apply the engineering design process. } \\
\text { - Explain the characteristics of effective team behavior } \\
\text { and the importance of teamwork in an engineering } \\
\text { environment. } \\
\text { - Collaborate effectively to solve problems, complete } \\
\text { projects, and present findings and results. } \\
\text { - Explain the interdisciplinary nature of solving } \\
\text { complex engineering problems. } \\
\text { - Demonstrate the global significance of specific } \\
\text { engineering applications. } \\
\text { - Communicate effectively through various mediums. } \\
\text { By the end of the semester students will have gained } \\
\text { experience in: } \\
\text { - The ability to apply knowledge of mathematics, } \\
\text { science, and engineering. } \\
\text { - The ability to identify, formulate, and solve } \\
\text { engineering problems. } \\
\text { - An understanding of professional and ethical } \\
\text { responsibility. } \\
\text { - The ability to communicate effectively. } \\
\text { - The broad education necessary to understand the } \\
\text { impact of engineering solutions in a global, economic, } \\
\text { environmental, and societal context. } \\
\text { - The recognition of the need for, and an ability to } \\
\text { engage in lifelong learning. } \\
\text { - The knowledge of contemporary issues. } \\
\text { These outcomes relate to ABET outcomes a, e, f, g, h, } \\
\text { i, and j. }\end{array}$ \\
\hline
\end{tabular}

Figure 1: Comparison of learning objectives and student outcomes prior to and after revising EGS1006L Introduction to the Engineering Profession. 


\begin{tabular}{|c|c|c|c|c|c|c|c|c|c|c|c|c|c|c|c|c|c|c|c|c|c|}
\hline 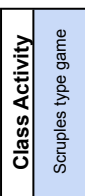 & 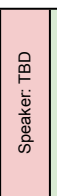 & & 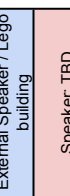 & 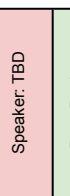 & 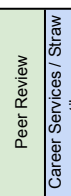 & & 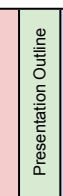 & & 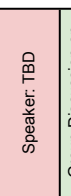 & 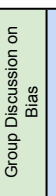 & 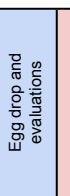 & 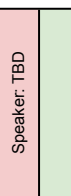 & & 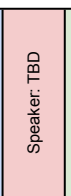 & 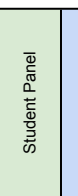 & & & 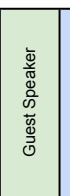 & 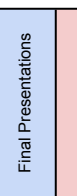 & 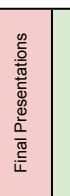 & 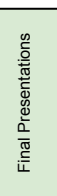 \\
\hline 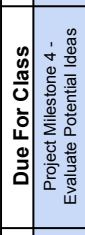 & 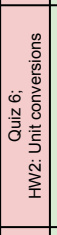 & & 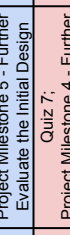 & 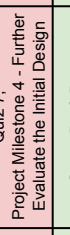 & 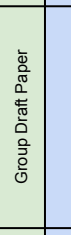 & 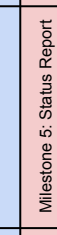 & 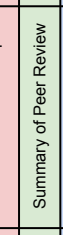 & 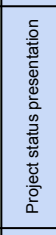 & $\begin{array}{l}\stackrel{\infty}{\tilde{z}} \\
\stackrel{\tilde{z}}{2}\end{array}$ & 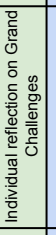 & 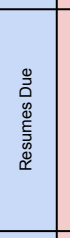 & 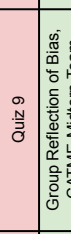 & 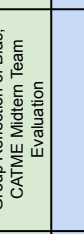 & $\begin{array}{l}\stackrel{\circ}{\bar{y}} \\
\frac{\bar{y}}{\sigma}\end{array}$ & 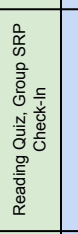 & & 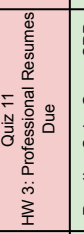 & 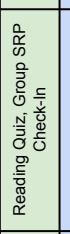 & & & \\
\hline 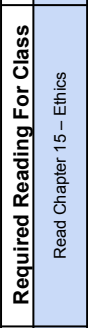 & 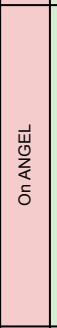 & & 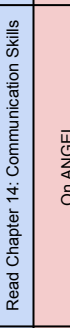 & 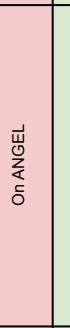 & $\mid$ & 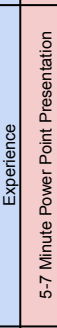 & 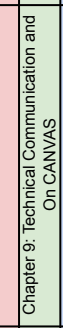 & & 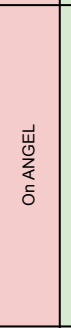 & 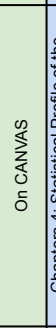 & 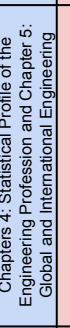 & 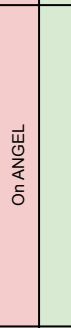 & & 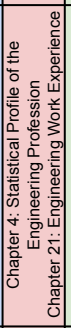 & 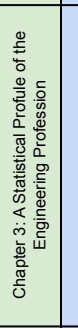 & & 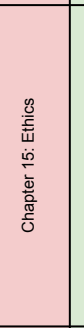 & 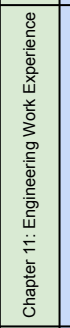 & & & \\
\hline 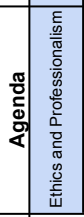 & 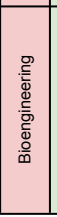 & 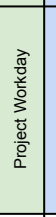 & 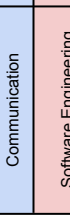 & 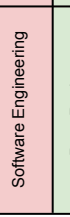 & 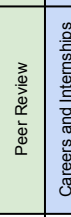 & 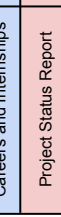 & 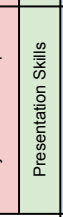 & 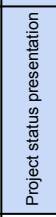 & 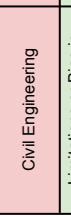 & 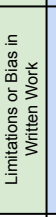 & 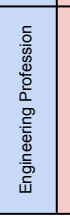 & 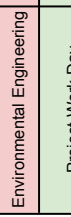 & 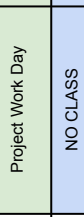 & 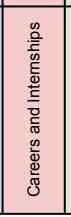 & 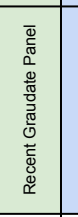 & 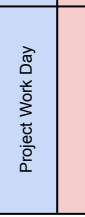 & 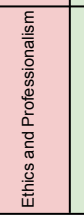 & 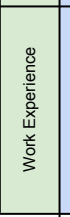 & 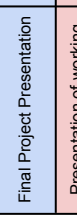 & 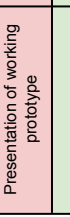 & 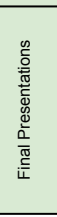 \\
\hline כ": & ه & & & 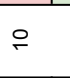 & & $F$ & & & $\simeq$ & & & m & & $\stackrel{\Delta}{\sim}$ & & & 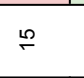 & & & $\stackrel{\circ}{-}$ & \\
\hline 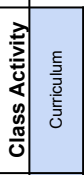 & 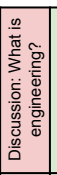 & & & 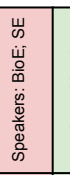 & 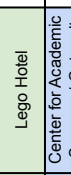 & 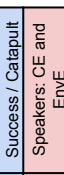 & & 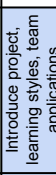 & 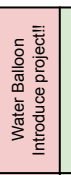 & 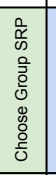 & 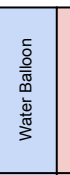 & 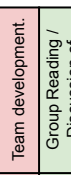 & 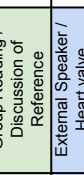 & 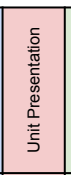 & 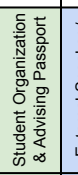 & 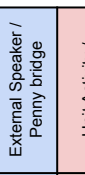 & 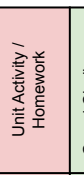 & 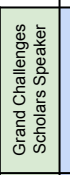 & 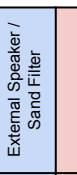 & 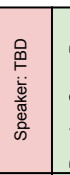 & 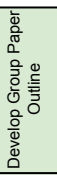 \\
\hline 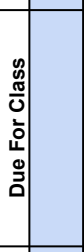 & & 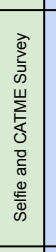 & 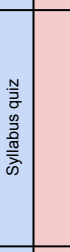 & & 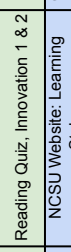 & 离 & 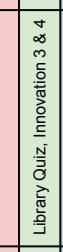 & & 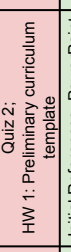 & & & 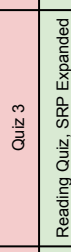 & 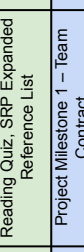 & 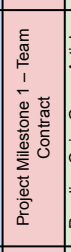 & 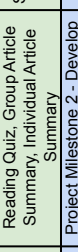 & 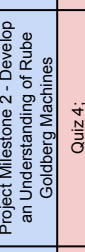 & 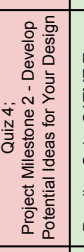 & 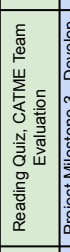 & $\begin{array}{ll} \\
0 \\
0 \\
0\end{array}$ & 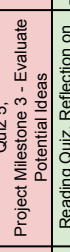 & 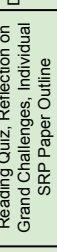 \\
\hline 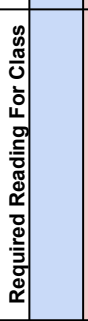 & & & 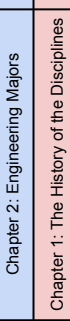 & 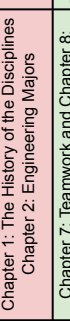 & 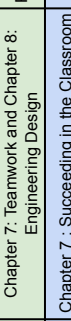 & 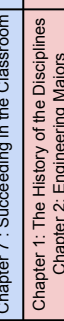 & & 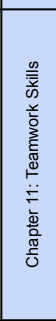 & 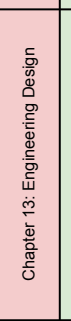 & & 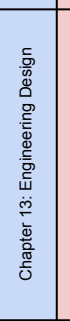 & 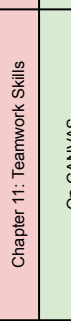 & 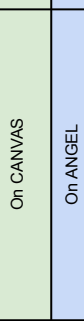 & 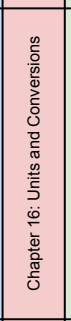 & 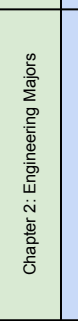 & 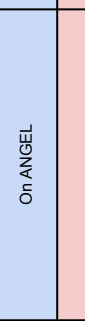 & 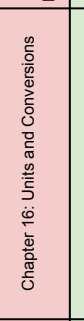 & 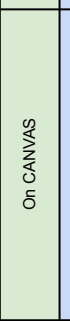 & 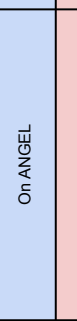 & 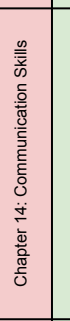 & 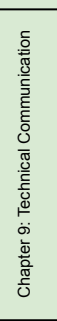 \\
\hline 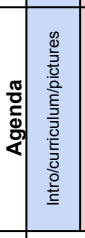 & 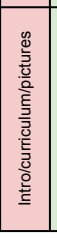 & 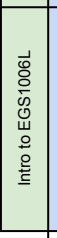 & 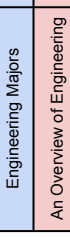 & 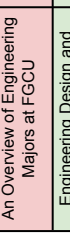 & 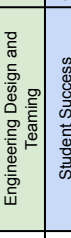 & 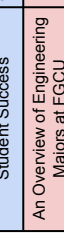 & & & 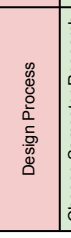 & 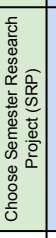 & $\begin{array}{l}\frac{0}{0} \\
\frac{8}{0} \\
\frac{0}{5} \\
\frac{6}{8} \\
0\end{array}$ & . & 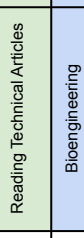 & $\overline{\frac{9}{\underline{\underline{s}}}}$ & 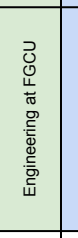 & 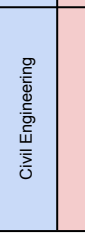 & $\begin{array}{l}\overline{\overline{\underline{w}}} \\
\frac{\underline{\underline{\underline{y}}}}{}\end{array}$ & 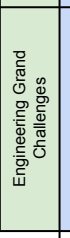 & 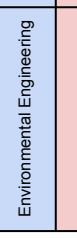 & 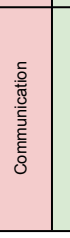 & 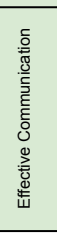 \\
\hline 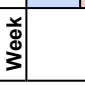 & - & & & $\sim$ & & $m$ & & & $\sigma$ & & & ๑ & & 0 & & & r & & & $\infty$ & \\
\hline
\end{tabular}

Figure 2: EGS1006L Agenda Before and After Course Revision. Comparison of topics, assignments, activities and deliverables for EGS1006L - Introduction to the Engineering Profession prior to and after the course revision. Blue, red and green highlighted cells indicate agendas from 2008, 2012 and 2014 respectively. 


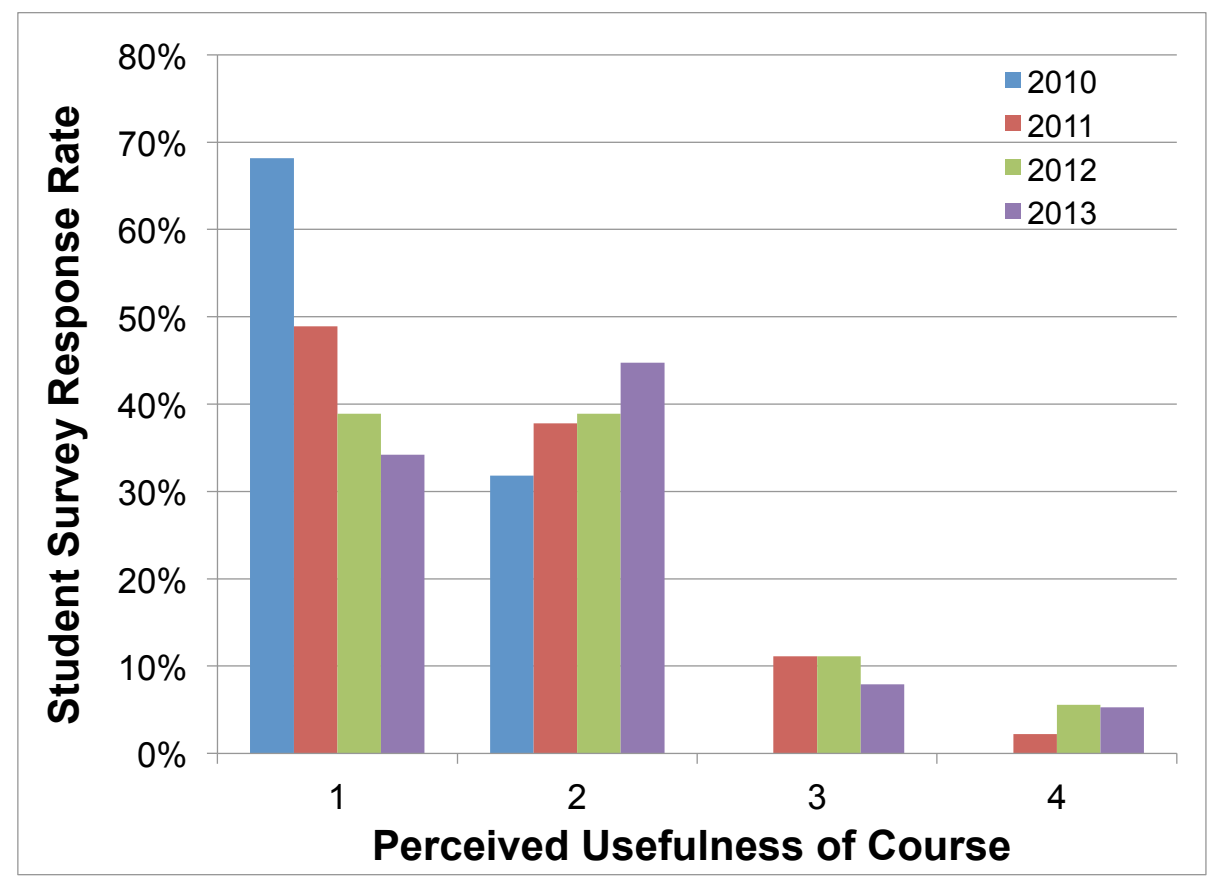

Figure 3: Senior Exit Survey Feedback Demonstrating the Need for Curricular Revision. Exit survey feedback for EGS 1006L - Introduction to the Engineering Profession. A score of 4 indicates the senior students felt the course was "most useful" while a score of 1 indicates "not useful".

Compounding these issues was the growth rate of the College. As enrollment grew within the College, additional sections of Introduction to the Engineering Profession were added to meet the additional demand. The number of sections per semester was increased from two to three, and total enrollment within the course ballooned from 90 students in per semester in 2009 ( 2 sections) to 157 students per semester in 2013 (3 sections). While the number of students per section increased only slightly ( 45 per section to 52 per section), the physical limitations of the classrooms and lack of additional support staff made activities difficult to coordinate.

Furthermore, the additional section placed added pressures on outside speakers, who were now asked to further extend their time commitment to the course, often spending $3+$ hours on campus on the days of their talks, repeating their message to the various sections. Ultimately, it was determined continuing to teach the course in this this manner was no longer feasible given the College's continued growth, forcing us to re-envision the course from the ground up.

\section{Course Revisions}

The authors and additional faculty teaching the course within the College first met in Fall 2013 to begin work on a complete revision / re-envisioning of this introductory course. The primary goal of the revised "Introduction to the Engineering Discipline" course was to create a one-credit hour learner-centered course with well-developed course objectives. Additionally, emphasis was placed on clearly tying all in-class and out of class activities to an overarching design project. In developing this course, the authors wanted to ensure that course expectations were in line with a one credit-hour course, yet still provide opportunities to discuss topics within and across the 
disciplines. Furthermore, we wanted to ensure that both the faculty and students used the single, weekly, 75-minute lesson meeting time effectively. What was ultimately developed was a course with an emphasis on information literacy, teamwork, and communication skills, focusing on engineering innovations related to the Grand Challenges.

Choosing to frame the revised course around the Grand Challenges was one of the first decisions we made in revising this course. The Grand Challenges Scholars Program at FGCU had been recently approved, and an additional opportunity to further integrate the Grand Challenges into the Engineering curriculum and promote the opportunity to encourage students to participate in the scholars program was seen to be a positive. Furthermore, the Grand Challenges offered a platform onto which almost any faculty across the College could integrate their areas of expertise to the class. However, because we wanted the projects to remain student-driven, we didn't want to immediately present the Grand Challenges to the students and force their research into a specific area. We wanted to regain the active learning component that had been lost in the previous version of the course over the years, but we wanted the active learning to be pertinent to the semester project, such that in-class and out of class activities were purposed and meaningful. Based on our own experiences in teaching upper-level courses, as well as input from our advisory board, we wanted these activities to focus on critical thinking, information literacy and technical writing. Lastly, since this course was still an introduction to the engineering profession, we needed to keep some of the information in the course related to engineering as a profession.

Based on these goals, we came up with a set of new learning objectives and student outcomes for the course, shown in Figure 1. Although the mechanics of the course are still very similar to how the previous version of the course was run; the activities, presentations, and overall course integration, as shown in Figure 2, are very different. This new approach, combined with the new learning outcomes, emphasize the development of skill sets necessary for future success both within our engineering program, something the previous course outcomes only peripherally addressed, as well as after graduation. Peripheral activities that did not significantly contribute to course deliverables were removed, and nearly all in-class and out-of-class activities were tied directly to the final project, which is a research paper and subsequent presentation of a recent innovation related to the student's field of study. As the course is still an introduction to the engineering profession, some of the classes, particularly toward the end of the semester were designed to better acquaint the students with resources within the College and University, particularly those that will benefit them not only as they progress through their degree programs, but also as they think about their future careers.

\section{Course Implementation - Fall 2014}

For Fall 2014, 103 students enrolled the course, divided over three sections. Although this number is less than the number of students in 2013, one program, Software Engineering, no longer requires this course as part of their curriculum. Factoring this into the previous numbers for 2013 fall enrollment, the numbers for fall 2014 were equivalent to that in 2013 (54 of the 157 
students from the 2013 data were Software Engineering Majors). Although there remained three sections of the course, all three sections were taught simultaneously, something that had not been previously attempted. This allowed for flexibility in scheduling classes, and offered unique opportunities to have classes meet in different ways. For example, students were allowed to sign up for any section they desired, although each section was taught by someone from each of the three programs within the College (Bioengineering, Civil Engineering and Environmental Engineering). However, when they were placed into groups for their semester projects, they were placed based on their major (teaming, and student evaluations within teams was done online using the CATME Smarter Teamwork system ${ }^{c}$ ). The decision to group students by major was based on the idea that the final projects would ultimately focus upon a current innovation within their own field and having students delve into the science and engineering behind transformative research in their intended field could potentially provide a greater appreciation for the scope of their specific major. Looking at Figure 2, lessons that require discipline specific information to be provided to the teams, or lessons that contain team-driven activities are organized such that students are separated by discipline. Alternatively, lessons that do not contain a team-driven component or do not require discipline specific information to be distributed are organized by sections, such that all three disciplines are present in the same room. Scheduling the multiple sections to run concurrently also allows all the sections to meet together in a larger classroom so that outside speakers can reach out to all the students at the same time.

Figure 2 also demonstrates the in-class and out of class activities that the students participated in for the revised course. Many of the activities and subsequent homework assignments were designed as milestones for successfully completing the semester project. In many ways, this course was designed in a similar fashion as one might design a capstone design course, with multiple deliverables contributing to an overall portfolio and final project. In addition to streamlining the course deliverables and in-class activities, we also lobbied for additional help in the classroom to provide as much feedback to the students as possible as they were working on their projects. Six TAs were recruited for the first semester of this revised course, with two students (one junior and one senior) from each of the three engineering disciplines. This provided three facilitators in each classroom (the faculty member, and two TAs), covering each of the disciplines on days where the class met by section and covering the same discipline on days where the class met in their research groups. By rotating the TAs and students across different faculty members, the students were able to initiate numerous interdisciplinary and disciplinary discussions not only with their peers, but also with the TAs and faculty members. By having one junior and one senior TA from each engineering discipline (this will expand to one senior and two juniors in future offerings), we hope to develop continuity from semester to semester and from year to year to limit the necessity of retraining new cohorts of TAs.

The basic layout of the course was to provide the students a means by which they would be able to successfully research and discuss a current innovation within their field of study by the end of the semester. While the formation of teams via CATME was being completed, each of the students on their own were asked to look through popular media and find multiple current

\footnotetext{
${ }^{\mathrm{c}}$ http://www.catme.org
} 
innovations within their proposed field of study. This was meant to make the students aware of the breadth of activity within their field, and hopefully peak their interest or confirm their interest in a particular field of engineering. During this time frame we also introduced the students to the University's STEM librarian, along with tools necessary to utilize the library to begin to more thoroughly research a particular subject. The students were then asked to pick their favorite innovation, and do some additional research into that particular innovation. At this point in time, the students were placed into groups of 4-5 students per group, and they were asked to make a presentation regarding their innovation, attempting to convince the group as to why their topic should be the group's research topic for the semester. Upon determining which topic the group wanted to investigate for the semester, students were introduced to a variety of topics and inclass activities that were designed to help them write a better paper and give a better final presentation, including topics on bias in writing, how to effectively read technical articles, effective communication, and presenting to a non-technical audience. They were also introduced to the Grand Challenges, and asked to categorize and explain their own research within the context of either the fourteen challenges, or the four overarching themes.

As the semester progressed, weekly deliverables were focused on the further development of the ultimate final deliverables of a paper and presentation on their chosen innovation. Paper outlines received feedback from the TAs during class, and initial draft paper submissions were anonymously distributed for peer feedback. Individuals evaluated the draft papers outside of class, and class time was dedicated to the aggregation of individual thoughts into a group evaluation, which was then anonymously provided back to the authors. In this way, students were asked not only to critically think about their own topic, but also about two other topics to which they often had limited exposure (as evaluations were arranged in a cross-disciplinary manner). Additionally, students gained exposure to the revisionary writing process for a technical paper - something many had experienced from a composition perspective, but not from the perspective of an engineering course.

Groups also developed presentation outlines, taking into consideration not only what was to be covered, but who would cover certain information, and the timing associated with each concept. Completed during the lesson on presenting to a non-technical audience, groups benefitted from TA and instructor feedback during the outline development, and were encouraged to remember that final presentations would be presented to an audience comprised of all engineering majors. During the final presentation time, all students were asked to individually assess group presentations based on a simplified Likert scale rating of the key components of the presentation grading rubric. Both the grading rubric (utilized by the instructor) and the Likert scale ratings (utilized by the students) were presented and available to students prior to the final presentation time period. 


\section{Assessment of Initial Offering - Fall 2014}

Although limited in scope compared to the ultimate level of desired assessment of the course, select assessment was performed on the initial Fall 2014 offering of the course. This assessment included both student surveys, as well as evaluation of student performance on course activities. Additionally, the initial assessment of the course provided a clearer idea of what we really wanted to consider and allowed us to refine our assessment instruments for implementation in future offerings of the course.

Student surveys took two separate forms. The first was a simple 5 question paper survey administered during the final presentation period. This survey contained 4 Likert-style questions each with a scale of 1 (low) to 5 (high). The first two questions asked about the students' interest in the course content, both before taking the course and at the conclusion of the course. The next two focused on the students' confidence in the topics covered in the course (again asking for the relative level prior to course enrollment and after completing the course). The final question asked for the students' major and how likely they were to remain in that major. Options on this final question included: remaining in major, definitely changing major (to what?), thinking about changing major (to what?), and still undeclared. The completion rate for this survey was $78 \%$ (n $=80$ of 103). Of these 80 students, $58(73 \%)$ responded that they intended to stay in their current major, $9(11 \%)$ responded that they were thinking about switching, $6(8 \%)$ stated that they were definitely switching, and 1 was still undeclared. Of those changing or thinking about changing, 6/15 (40\%) listed another engineering major, 5 (33\%) listed a science, 2 listed business and 2 were undecided.

Pre and post interest in the course content is shown in Figure 4. Although the average interest in the course did not significantly change from the pre-test to the post-test, those that expressed the highest interest in the course material increased by $12.5 \%$ over the course of the semester. Likewise, as shown in Figure 5, there was an observed increase in the overall confidence of the students, with the average increasing from 3.67 in the pre-test to 4.33 in the post-test.

A second survey was conducted online utilizing the "Student Assessment of their Learning

Gains" (SALG) website ${ }^{d}$. Due to the relatively low return rate from the fall semester $(10 \%)$, this assessment will be run again in the spring to improve the overall number of subjects surveyed. It is the authors' belief that a delay in the IRB protocol approval, which prevented the SALG survey from being administered until the last two weeks of the semester, could be an influencing factor on the low return rates. Since approval is already in place for the spring offering, requests for survey completion will be sent to the students earlier in the semester, with the hope that an extended time period will result in greater participation. If the online version still results in lower than desired completion rates consideration will be taken to implement a paper version of the survey during class in the Fall 2015 offering of the course.

${ }^{d}$ http://www.salgsite.org 


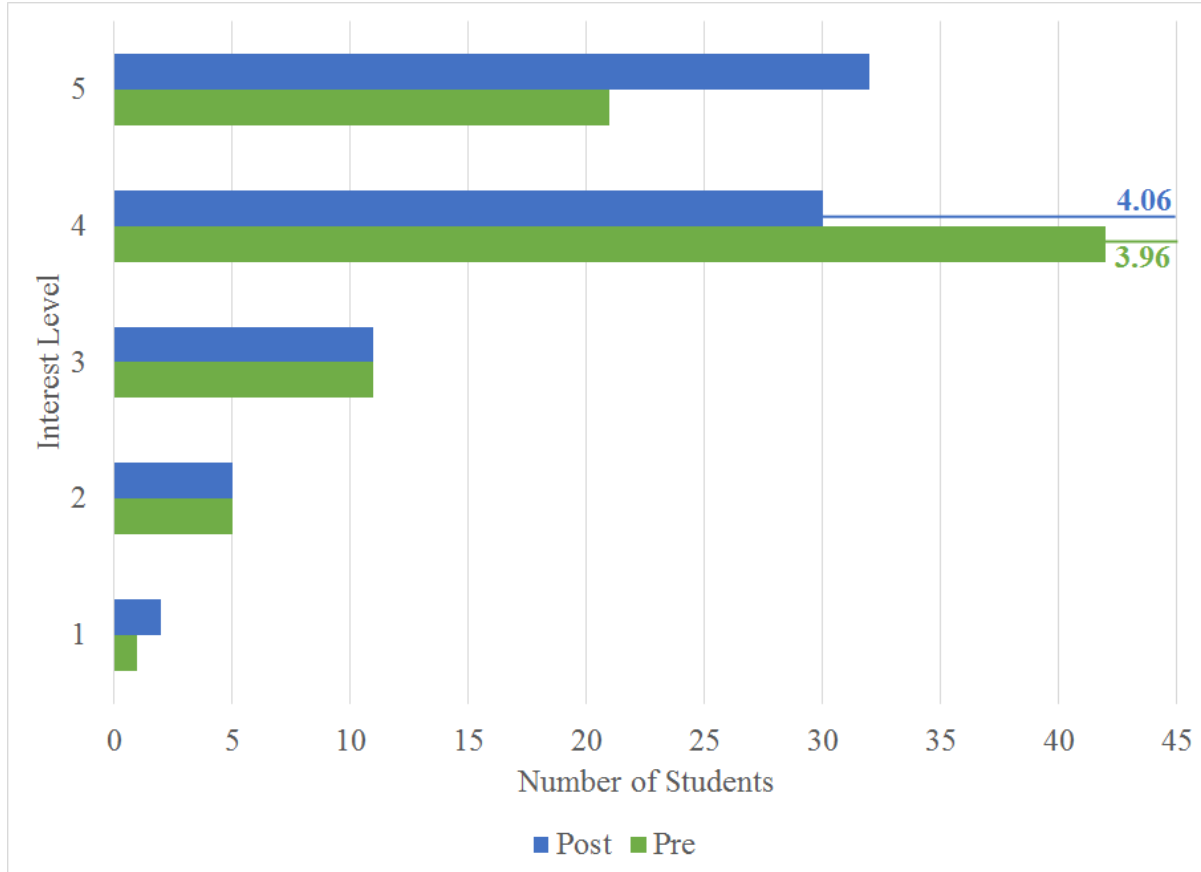

Figure 4: Results of Student Survey of Interest Pre and Post Course. Survey results in response to the question of "What was / is your interest in the course content?" where 1 indicates a low interest and 5 indicates a high interest. Average values pre and post are presented as numbers and thin lines.

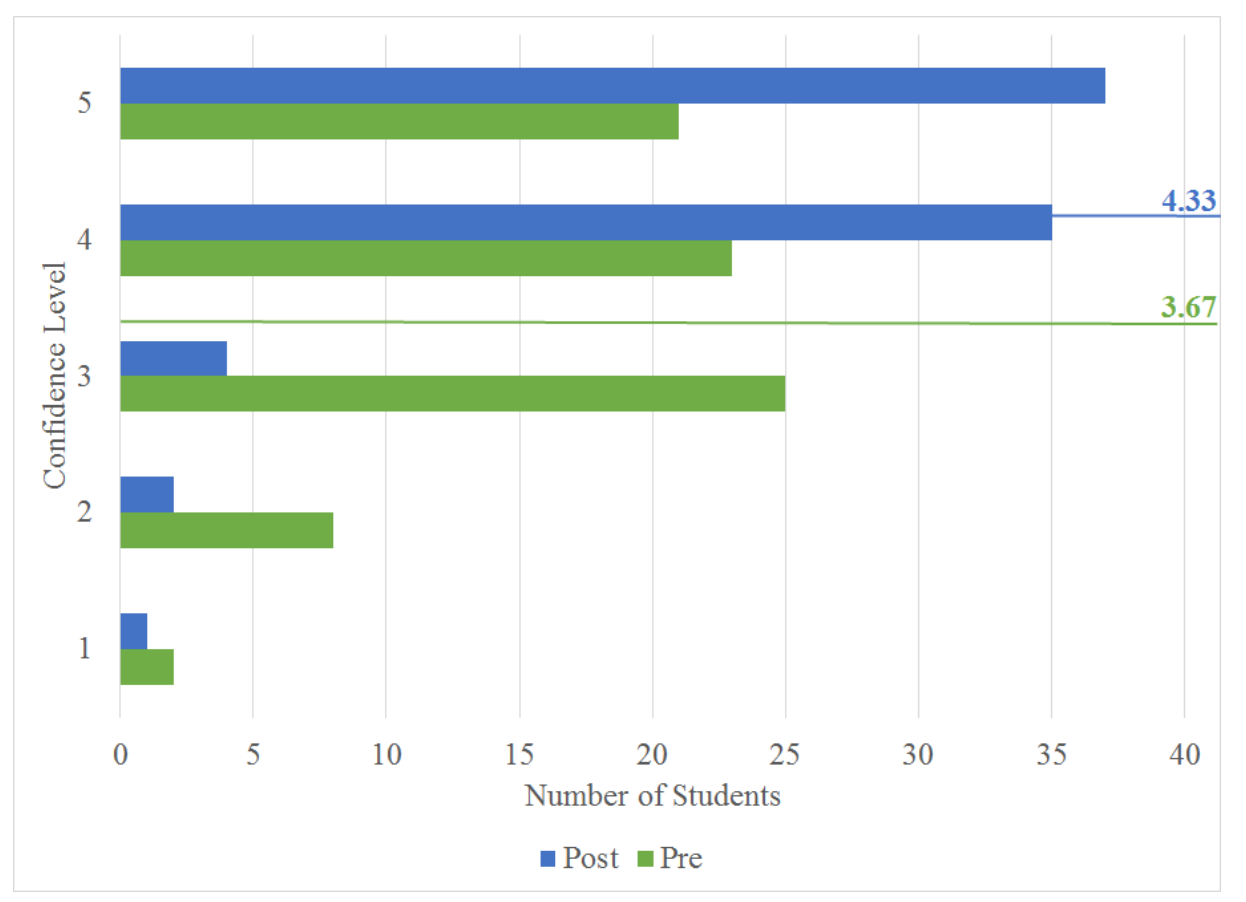

Figure 5: Results of Student Survey of Confidence Pre and Post Course. Survey results in response to the question of "What was / is your confidence level with this course content?" where 1 indicates a low confidence and 5 indicates a high confidence. Average values pre and post are presented as numbers and thin lines. 
In addition to student surveys, evaluation of student performance on both the final papers and final presentations occurred. Performance was measured against established rubrics that were presented to the students early in the semester and are shown in Tables 1 and 2. These rubrics were designed to include sections focusing on points discussed in the course throughout the semester - e.g. APA formatting, scholarly references, effective communication, the NAE Grand Challenges, etc. Students were encouraged to consult these rubrics starting as early as the outline stage, and continuing through draft and final submission preparation.

\begin{tabular}{|c|c|c|}
\hline Criteria & Longer Description & Points \\
\hline $\begin{array}{l}\text { Overall } \\
\text { Organization }\end{array}$ & $\begin{array}{l}\text { Has appropriate sections that flow logically, provide sufficient topic coverage, and } \\
\text { are representative of current body of knowledge. Uses scientific language and } \\
\text { structure throughout. }\end{array}$ & 10 \\
\hline Introduction & $\begin{array}{l}\text { Has a concise statement of purpose (needs statement) and problem description } \\
\text { ("hook"). Provides direction and briefly outlines paper. Provides evidence of } \\
\text { societal impact and relationship to Grand Challenges where appropriate ("big } \\
\text { picture"). }\end{array}$ & 10 \\
\hline $\begin{array}{l}\text { General } \\
\text { Discussion }\end{array}$ & $\begin{array}{l}\text { Writing clearly illustrates a logical approach to the subject. Includes historical } \\
\text { background, current status and future direction of topic. Clearly presents topic with } \\
\text { a discussion of various analyses in a factual manner. Integrates results from } \\
\text { multiple sources in a smooth manner. Provides a conclusion that concisely } \\
\text { summarizes key points without providing new information. }\end{array}$ & 40 \\
\hline References & $\begin{array}{l}\text { Uses primarily scholarly publications. Appropriately cites work within document } \\
\text { and correctly formats reference section to follow APA guidelines. }\end{array}$ & 20 \\
\hline Grammar & $\begin{array}{l}\text { Few, if any, spelling / grammar issues }(<3) \text {. Few, if any, errors in voice \& tense } \\
(<3) \text {. Always uses appropriate terminology. }\end{array}$ & 10 \\
\hline $\begin{array}{l}\text { Figures, } \\
\text { Tables \& } \\
\text { Equations }\end{array}$ & $\begin{array}{l}\text { Tables, graphs and figures correctly labeled, titled, and explained so read } \\
\text { understands full content of image without referring to text. Equations provided } \\
\text { where appropriate, correctly labeled, and all variables clearly explained. All } \\
\text { equations, figures, and tables referenced within written work in appropriate } \\
\text { location. }\end{array}$ & 10 \\
\hline \multicolumn{2}{|c|}{$\begin{array}{rr}\text { Total Points } \\
\end{array}$} & 100 \\
\hline
\end{tabular}

Table 1: Final Paper Rubric. This rubric includes the criteria, general description, and point values associated with the final paper submission. Papers were evaluated against this standardized rubric. 


\begin{tabular}{|c|c|c|}
\hline Criteria & Longer Description & Points \\
\hline $\begin{array}{l}\text { Overall } \\
\text { Organization }\end{array}$ & $\begin{array}{l}\text { Has a clear opening statement, catches audience interest, remains focused } \\
\text { throughout with smooth transitions, and includes a conclusion that ties together all } \\
\text { information. Falls within the } 7 \text { minute time range. Provides information in a factual } \\
\text { manner that is comprehensible to individuals with limited background knowledge. } \\
\text { Provides appropriate references. }\end{array}$ & 20 \\
\hline Content & $\begin{array}{l}\text { Provides concise explanation with focus on key elements in a factual manner } \\
\text { without appearing choppy. Includes historical context, contemporary findings, and } \\
\text { future direction of work and an integration of multiple sources. Uses scientific } \\
\text { language and appropriate terminology and provides definitions and explanations } \\
\text { where appropriate. }\end{array}$ & 50 \\
\hline $\begin{array}{l}\text { Technology } \\
\text { Use }\end{array}$ & $\begin{array}{l}\text { Utilizes a format that optimizes time, provides variety while minimizing } \\
\text { distractions, maximizes critical components, and effectively balances all included } \\
\text { components. Effectively implements selected technology resulting in few (if any) } \\
\text { visual errors (e.g. spelling/grammar errors, fuzzy pictures/graphs, blurry/pixelated } \\
\text { videos). Ensures visuals are appropriate relative to the size of the presentation } \\
\text { location. If employed, applies music, sound, voice-over, etc. in an appropriate } \\
\text { manner (e.g. language, volume, transitions, terminology, song selection). }\end{array}$ & 30 \\
\hline & Total Points & 100 \\
\hline
\end{tabular}

Table 2: Final Presentation Rubric. This rubric includes the criteria, general description, and point values associated with the final presentation. Student group presentations were evaluated against this standardized rubric.

With regards to paper submissions, students were considered to have met expectations if they earned a $70 \%$ or above in each individual category. Students earning an $85 \%$ or better in a category were considered to have exceeded expectations. Results of these thresholds are presented in Figure 6. Figure 7 presents the results of student performance against the final presentation rubric. For these submissions students were again considered to have met expectations if a $70 \%$ or better was earned, while exceeding expectations in the oral presentations required the achievement of a $90 \%$ or better in the category. Categories that do not sum to $100 \%$ indicate that all students in the course did not meet these specific objectives. Since this is the first offering of the first course in our engineering curriculum, having less than $100 \%$ of the students meet or exceed expectation provides evidence for potential areas of additional focus in future offerings of the course, as well as additional points of emphasis for courses later in the individual engineering programs. 


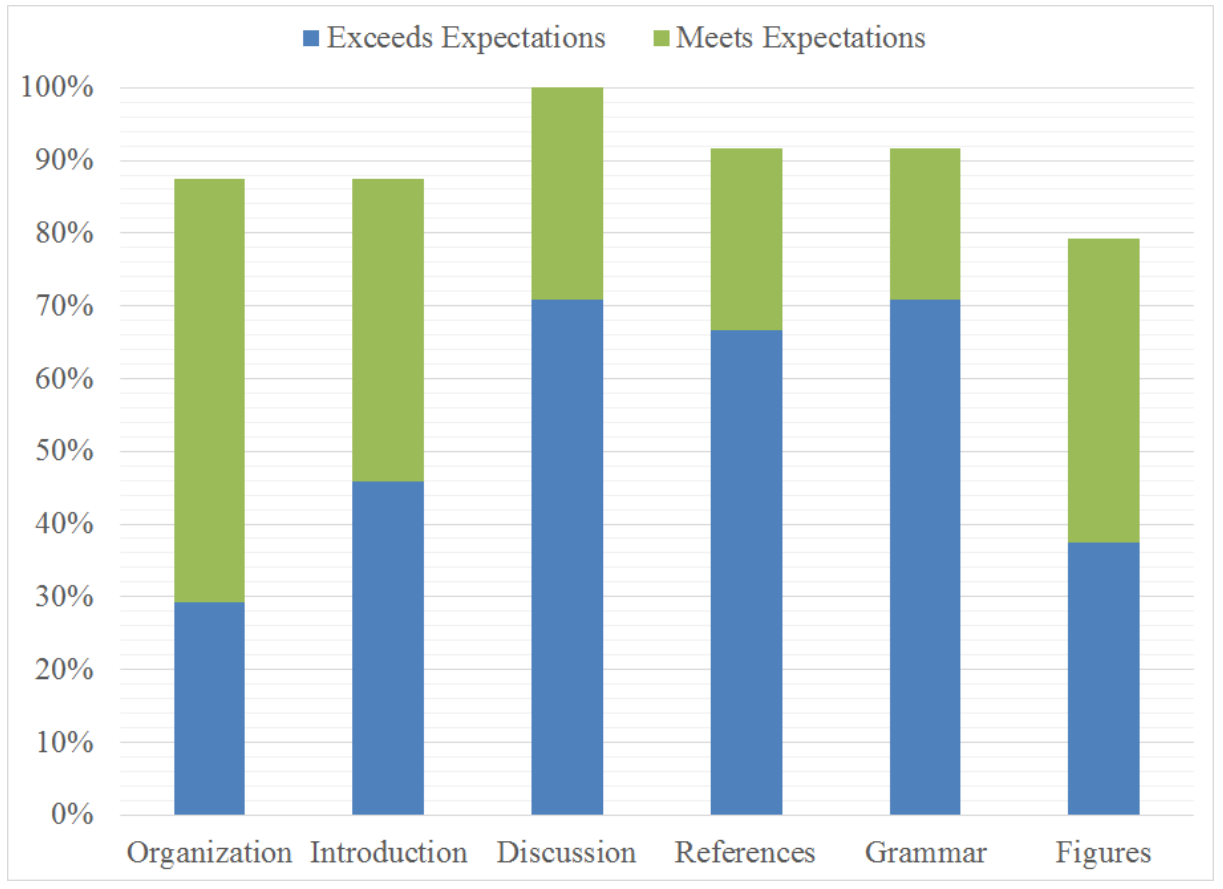

Figure 6: Student Performance on Final Paper Submission. This graph illustrates the achievement levels for each of the criteria on the final paper rubric. Students meeting expectations earned at least a $70 \%$ in the category, while students exceeding expectations earned at least an $85 \%$ in the category.

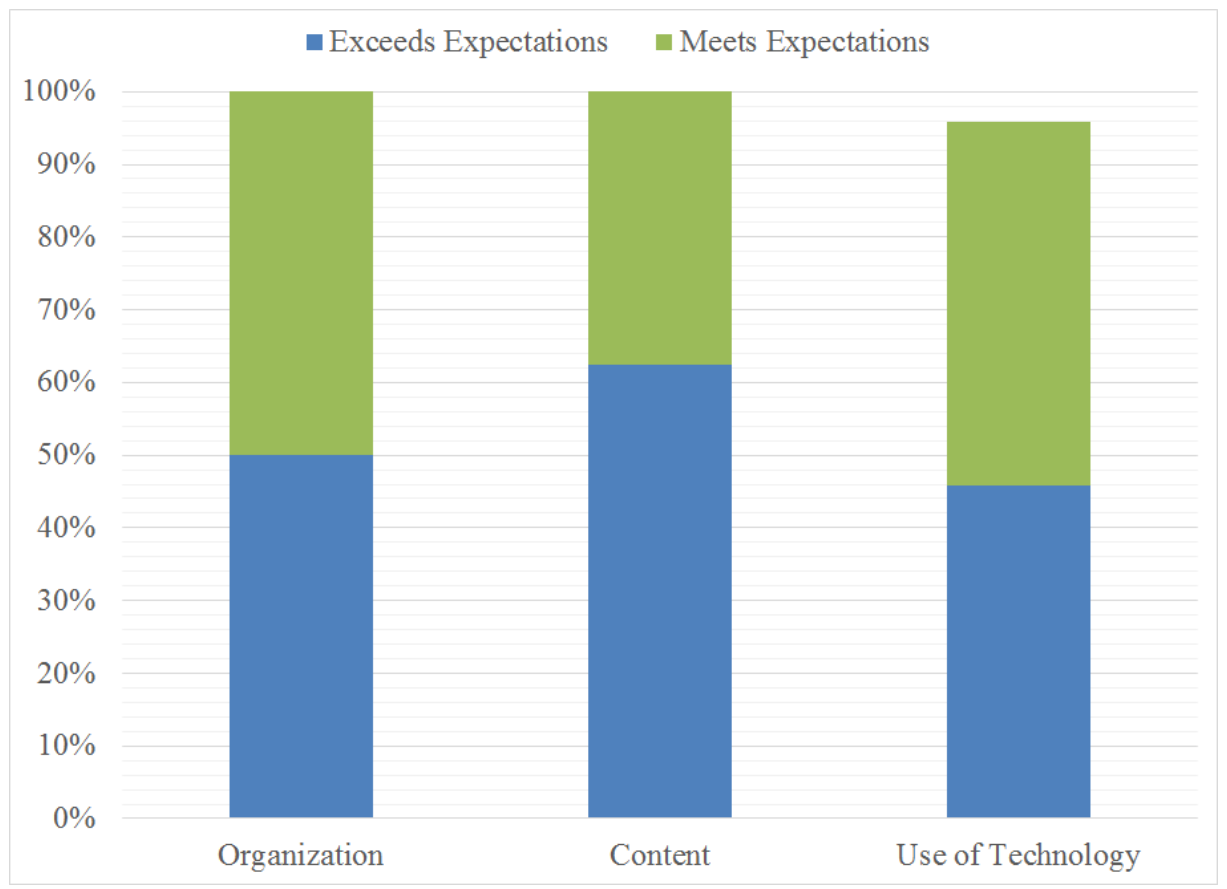

Figure 7: Student Performance on Final Presentations. This graph illustrates achievement levels for each of the criteria on the final presentation rubric. Students meeting expectations earned at least a $70 \%$ in the category, while students exceeding expectations earned at least a $90 \%$ in the category. 


\section{Revisions to Future Offerings - Spring 2015}

Although the general feedback and instructor evaluation of the course was positive, several clear areas existed where adjustments and improvements could be made. As the course is offered in both the fall and spring semesters, we took the opportunity to make some of the more critical, as well as some of the "quick and easy," adjustments for the Spring 2015 offering of the course. One major change was that of the grading scheme of the course. Two main adjustments were made to course grading. The first was in response to our recognizing that a large percent of the students' grades $(40 \%)$ remained out until the final exam period. The second stemmed from lower attendance rates as the semester progressed. When the course was initially developed for the fall of 2014, both of these items were discussed. It was originally thought that a weight of $30 \%$ for the final paper and $10 \%$ for the presentation was a reasonable division of the expected workload / time commitment for the course. Deliverables throughout the semester that "fed" into these final submissions were classified as either group assignments (15\% of the final grade) or individual assignments ( $25 \%$ of the final grade). The remaining $20 \%$ of the grade was associated with individual quizzes based on out of class reading assignments. After some discussion, the weight of the final paper was reduced to $25 \%$. Lower values were discussed, but ultimately we felt it was important to maintain the emphasis on this submission as a key component within the course. The percentages for the final presentation, group assignments, and individual assignments remained the same. The individual quiz percentage was reduced to $17 \%$, and the $8 \%$ gained from these two reductions was assigned to attendance. In the original discussion it was decided not to have an attendance policy for the course, however this was identified as an issue after the first semester. Since the course meets only once a week, checking in with groups on their progress was critical. Without the attendance, about a third of the groups chose to complete assignments without coming to class. While not inherently problematic, the reduced attendance did reduce the opportunity for near peer interaction with the TAs, informal feedback from the instructors, and general comments more easily conveyed in person versus via electronic means.

The second change from the fall to spring was that of providing additional feedback to groups over the course of the semester. Aside from the informal feedback from instructors and TAs during class and the draft feedback from peers, groups received little formal technical feedback on their submissions. The spring offering will incorporate a second round of peer to peer feedback, as well as more formal feedback from both TAs and Instructors at multiple points during the semester (e.g. topic selection, outline, draft stages).

A more intentional multi-disciplinary integration was the third change implemented. Although this was the original intent with the simultaneous classes, the actual delivery of the first offering was biased toward discipline specific classes - with only 5 of the 16 weeks integrating disciplines. Upon completion of the fall semester we took a closer look at the course topics, and have adjusted the schedule so that 11 of the 16 weeks will involve cross disciplinary interactions. The schedule topics themselves did not change, we are simply capitalizing on the opportunity to create multi-disciplinary discussions in addition to discipline focused conversations. 
The final adjustment was more logistical in nature, and falls into the "quick and easy" category of fixes to the course. Short PowerPoint presentations were developed for each class presenting the discussion questions for the lesson, detailing the in class activity deliverables, and providing a closing slide that would "look ahead" and provide housekeeping details for the upcoming week. In the first offering these items were all in separate locations within the course management system, and pulled up by instructors as needed. The PowerPoint allows for a cleaner and more concise presentation of the material, and provides an additional tool to better assure the level of instruction is equal across multiple sections and semesters.

\section{Conclusion}

Overall, the format change associated with this introductory course has been well-received and liked by both faculty and students within the College. Many of the TAs who took this course under the old format have commented to us as to how they are envious of the current students, as the new course not only provides an opportunity to develop information literacy, critical thinking and writing skills much earlier in the engineering curriculum, but it also provides them a more realistic expectation of what to expect in future engineering classes. While the current course assessment does not allow for a comparison between the previous and revised course offering (as it was not administered to the earlier version of the course), we plan to conduct a longitudinal comparison of student perception of course usefulness on the senior exit survey. In addition, although this course was redesigned to address perceived deficiencies in our current curriculum, focusing this course on information literacy, critical thinking and writing is timely given the focus of our next 5-year University QEP. In keeping this course as an interdisciplinary offering, we have some unique opportunities in regards to assessment of learning gains, both within the class and longitudinally, and will continue to use the Grand Challenges as a backdrop for the course. In regards to longitudinal assessment, we anticipate being able to follow these students throughout the remainder of their engineering career at FGCU, looking at additional metrics of learning gains in an effort to determine the impact of this new course format in the student's overall ability to collect, analyze and synthesize pertinent information within their field of study.

\section{References:}

1. Kuh G. High Impact Educational Practices: What They Are, Who Has Access to Them, and Why They Matter. Association of American Colleges and Universities; 2008.

2. Blanchard S, Egiebor N, Sweeney J, et al. Blank Slate Engineering at Florida Gulf Coast University-Innovative and Multidisciplinary from the Ground Up. In: Proceedings of the 2006 ASEE Annual Conference and Exposition.; 2006.

3. National Academy of Engineering. Educating the Engineer of 2020: Adapting Engineering Education to the New Century.; 2005. http://www.nap.edu/catalog.php?record_id=11338. Accessed January 29, 2014. 
4. National Academy of Engineering. The Engineer of 2020: Visions of Engineering in the New Century.; 2004. http://www.nap.edu/catalog.php?record_id=10999. Accessed January 29, 2014.

5. Beichner RJ, Saul JM, Allain RJ, Deardorff DL, Abbott DS. Promoting collaborative groups in large enrollment courses. In: Proceedings of the 2000 ASEE Annual Conference and Exposition.; 2000. 